\title{
Collective electromagnetic emission from molecular layers on metal nanostructures mediated by surface plasmons
}

\author{
V. Giannini, ${ }^{1}$ J. A. Sánchez-Gil,, ,* J. V. García-Ramos, ${ }^{1}$ and E. R. Méndez ${ }^{2}$ \\ ${ }^{1}$ Instituto de Estructura de la Materia, Consejo Superior de Investigaciones Científicas, Serrano 121, 28006 Madrid, Spain \\ ${ }^{2}$ Departamento de Óptica, División de Física Aplicada, Centro de Investigación Científica y de Educación Superior de Ensenada, \\ Ensenada, Baja California 22800, Mexico \\ (Received 26 April 2007; published 28 June 2007)
}

\begin{abstract}
Collective electromagnetic processes stemming from molecular emission close to complex nanostructured metal surfaces pumped at and/or near surface-plasmon resonances are theoretically investigated. A classical electrodynamics model is used to describe macroscopically the surface molecular layer emission. Generalized Fresnel coefficients are analytically obtained for planar surfaces, indeed predicting collective quenching for redshifted emission at given angles. The model is introduced into a scattering formulation based on surface integral equations in order to explore collective spontaneous emission near metallic nanoantennas and surfaceenhanced Raman scattering. Frequency-shifted near-field patterns and properly defined enhancement factors are obtained that manifest collective processes and cannot be simply inferred from calculations of near fields at the pump frequency.
\end{abstract}

DOI: 10.1103/PhysRevB.75.235447

PACS number(s): 73.20.Mf, 78.30.-j, 78.67.Bf, 42.25.Fx

\section{INTRODUCTION}

In recent years, a variety of phenomena have been investigated involving electromagnetic (EM) radiation emitted close to complex nanostructured metal surfaces. ${ }^{1-16}$ To a large extent, the renewed interest stems from the fact that many such phenomena are mediated by localized surfaceplasmon resonances (LSPR) (due to collective excitations of the metal electron plasma), which give rise to large electricfield enhancements deeply modifying both the driving and the emitted electromagnetic fields (cf. Refs. 8, 10, and 14 and references therein). In a search for stronger, more selective LSPR, nanoparticles of increasingly complex shape (triangles, rectangles, stars, and dimers) are the subject of active investigation. ${ }^{6} 17-26$ Indeed, the term nanoantenna has been introduced to stress the ability of such nanoparticles to modify light emission both qualitatively and quantitatively. ${ }^{27}$ Several LSPR-mediated, light-emitting processes from atoms, molecules, or solid films close to nanostructured metals are known to have implications in nanophotonics and (bio)sensing. Among them is surface-enhanced Raman scattering (SERS): As far as the electromagnetic enhancement mechanism is concerned, this is a subject of active research due to advances as single molecule detection and controllable substrates. ${ }^{6,14-16}$

In general, in order to accurately describe the entire process, several complex theoretical steps are required. On the one hand, a rigorous theoretical formulation is necessary to account for the complex interaction between the incident field and the scattering configuration, which has a profound influence on the resulting driving EM fields. On the other hand, collective models of the EM emission from surface molecular layers (or any other surface light-emitting process) that go beyond the single dipole emission ought to be used. ${ }^{8,28,29}$ In this paper, we investigate theoretically the emission and scattering of classical electromagnetic radiation from a molecular layer on nanostructured surfaces, with special interest on accounting not only for the near EM field scattered at the pump frequency but also for the collective radiation processes at the (shifted) frequency of emission. The polarization vector accounting for the collective emission is presented in Sec. II, along with the analysis of the results it yields in the simple case of a planar surface. Section III briefly describes how such model is implemented in a rigorous scattering theory. In Sec. IV, numerical results based on the latter formulation are shown in two relevant cases: collective spontaneous emission from metal nanoantennas and SERS on nanostructured metal surfaces. The concluding remarks are included in Sec. V.

\section{COLLECTIVE MODEL: POLARIZATION VECTOR}

A molecular layer is placed on the interface $S$ separating two dielectric media. We assume that the surface polarization vector within the undepleted pump approximation is given by

$$
\mathbf{P}(\mathbf{r}, \omega)=\delta(\mathbf{r}-\mathbf{R}) \chi_{\mathbf{s}}\left(\mathbf{R}, \omega, \omega_{0}\right) \mathbf{E}\left(\mathbf{R}, \omega_{0}\right),
$$

where $\mathbf{E}\left(\mathbf{R}, \omega_{0}\right)$ is the electric field on the interface $\mathbf{R} \in S$ at the pump frequency $\omega_{0}$. We introduce a $\delta$-function dependence in Eq. (1) to describe the fact that the molecular layer emission is basically a surface process from the standpoint of macroscopic classical electrodynamics. The microscopic details of the molecular emission at frequency $\omega$ are represented by a macroscopic surface susceptibility tensor $\chi_{\mathbf{s}}$, the dimensionality of which must be consistent with its surface character. ${ }^{30}$ The above surface polarization vector modifies the continuity conditions at the emission frequency, which now depend on the surface electric field at the pump frequency. By integrating Maxwell's equations across the interface, ${ }^{31}$ the tangential EM fields are modified in this manner:

$$
\mathbf{n} \times \Delta \mathbf{H}(\omega)=4 \pi i \frac{\omega}{c} \mathbf{n} \times \mathbf{n} \times\left[\chi_{\mathrm{s}} \mathbf{E}\left(\omega_{0}\right)\right],
$$


(a)
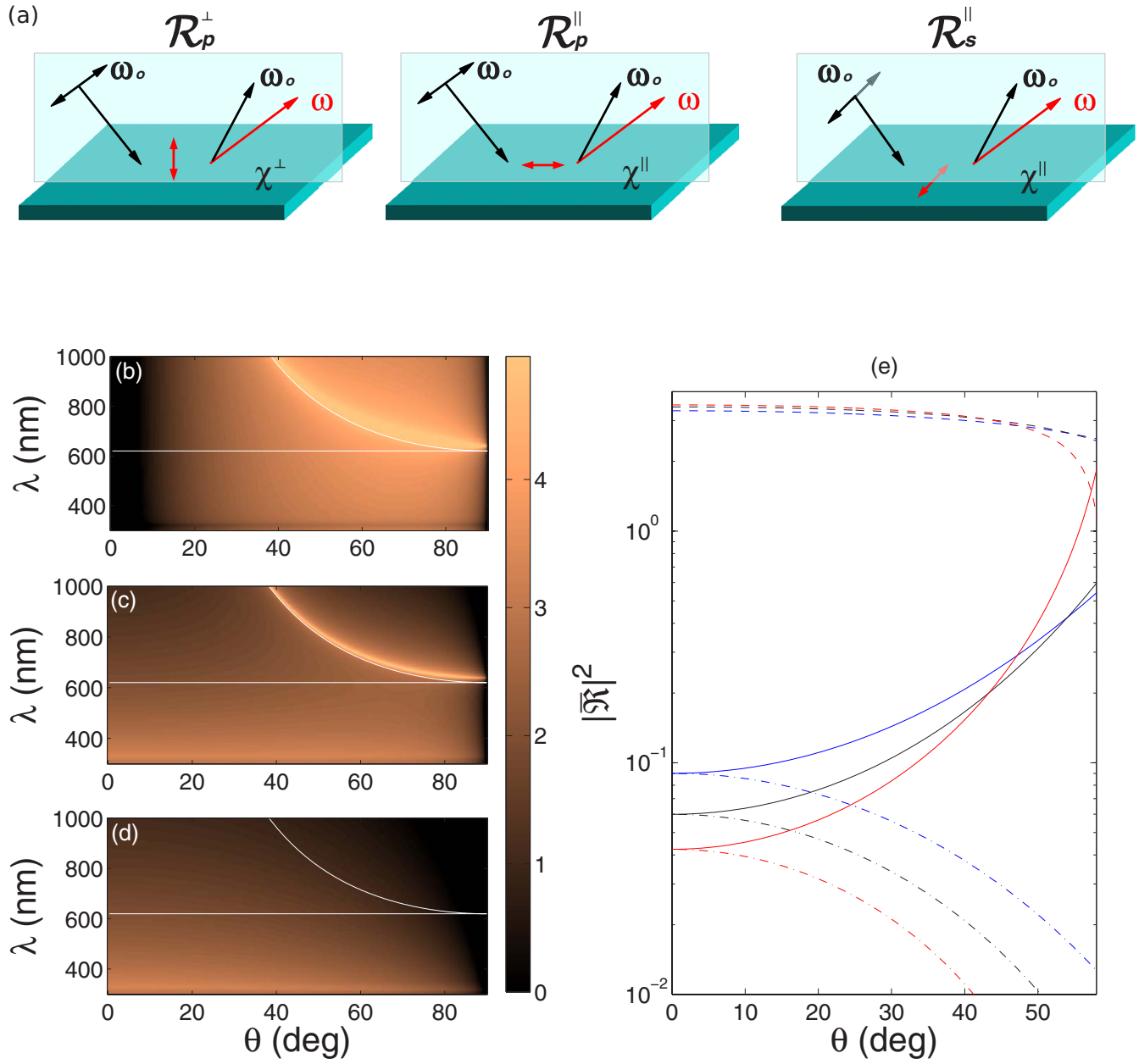

FIG. 1. (Color online) Square amplitude of the effective Fresnel coefficients [defined as depicted in (a)] for the collective surface molecular emission from a planar Ag surface as a function of both the pump angle of incidence $\theta_{0}$ and the emission wavelength $\lambda$ $=2 \pi c / \omega$, for a pump plane wave at $\lambda_{0}=2 \pi c / \omega_{0}=620 \mathrm{~nm}$. (b) $\log _{10}\left|\mathbb{R}_{p}^{\perp}\right|^{2}$, (c) $\log _{10}\left|\mathbb{R}_{p}^{\|}\right|^{2}$, and (d) $\log _{10}\left|\mathbb{R}_{s}^{\|}\right|^{2}$. (e) Angular dependence for fixed emission frequency of $\left|\bar{R}_{p}^{\perp}\right|^{2}$ (dashed curves), $\left|\overline{\mathbb{R}}_{p}^{\|}\right|^{2}$ (solid curves), and $\left|\overline{\mathbb{R}}_{s}^{\|}\right|^{2}$ (dash-dotted curves): $\lambda=\lambda_{0}$ (black), $\lambda=720 \mathrm{~nm}$ (red), and $\lambda$ $=520 \mathrm{~nm}$ (blue).

$$
\mathbf{n} \times \Delta \mathbf{E}(\omega)=-4 \pi \mu\left(\omega, \omega_{0}\right)(\mathbf{n} \times \nabla)\left[\mathbf{n} \cdot \varepsilon_{1} \chi_{\mathbf{s}} \mathbf{E}\left(\omega_{0}\right)\right],
$$

where $\quad \mu\left(\omega, \omega_{0}\right)=\left[2 \varepsilon_{1}(\omega) \varepsilon_{1}\left(\omega_{0}\right)\right]^{-1}+\left[2 \varepsilon_{2}(\omega) \varepsilon_{2}\left(\omega_{0}\right)\right]^{-1}$ $\left[\varepsilon_{1,2}(\omega)\right.$ being the dielectric functions of either media] and $\mathbf{n}$ is the normal to the surface $S$. In Eqs. (2), $\mathbf{E}\left(\omega_{0}\right)$ must be evaluated on the surface from medium 1.

Let us now determine what is expected from this model for a plane surface separating the vacuum $\left(\varepsilon_{1}=1\right)$ from a semi-infinite medium characterized by an isotropic $\varepsilon_{2}$ $=\varepsilon(\omega)$. For the sake of clarity, the surface susceptibility of the molecular layer is assumed to be diagonal, with only two nonzero components along the normal and tangential to the surface, that, is $\chi_{s}^{\perp}$ and $\chi_{s}^{\|}$, respectively. We assume for the moment that the emission is coherent (i.e., that there is a deterministic relation between the phase of the pump beam and the emitted field at the shifted frequency). In the case of a harmonic plane wave (pump beam at $\omega_{0}$ ) incident at an angle $\theta_{0}$ on a planar surface with amplitude of 1 , we define the effective Fresnel coefficients for the entire emission pro- cess as the amplitude of the field emitted by the molecular layer at $\omega$ [see Fig. $1(\mathrm{a})]$ : $\mathbb{R}_{s}^{\|}\left(\theta_{0}, \omega_{0}, \omega\right)$ for $s$ polarization and $\mathbb{R}_{p}^{\|}\left(\theta_{0}, \omega_{0}, \omega\right)$ and $\mathbb{R}_{p}^{\perp}\left(\theta_{0}, \omega_{0}, \omega\right)$ for $p$ polarization from, respectively, the $\|$ and $\perp$ components of the diagonal surface susceptibility tensor $\chi_{s}$. The scattering angle $\theta$ is imposed by transverse momentum conservation: $K_{0}=\left(\omega_{0} / c\right) \sin \theta_{0}$ $=(\omega / c) \sin \theta=K$. By imposing the continuity conditions [Eqs. (2)] at $\omega$ with the pump surface electric field at $\omega_{0}$ given in terms of the well-known Fresnel coefficients $R_{p}$ and $R_{s}$, it is straightforward to obtain the effective Fresnel coefficients for the collective emission:

$$
\begin{aligned}
\mathbb{R}_{p}\left(\theta_{0}, \omega_{0}, \omega\right) \equiv & \mathbb{R}_{p}^{\|}\left(\theta_{0}, \omega_{0}, \omega\right)+\mathbb{R}_{p}^{\perp}\left(\theta_{0}, \omega_{0}, \omega\right) \\
= & -i \frac{\omega}{\omega_{0}}\left\{\frac{q_{0}^{>} q^{<}\left[1-R_{p}\left(\theta_{0}, \omega_{0}\right)\right]}{\varepsilon(\omega) q^{>}+q^{<}} \chi_{s}^{\|}\right. \\
& \left.-\frac{\varepsilon(\omega) \mu\left(\omega, \omega_{0}\right) K^{2}\left[1+R_{p}\left(\theta_{0}, \omega_{0}\right)\right]}{\varepsilon(\omega) q^{>}+q^{<}} \chi_{s}^{\perp}\right\},
\end{aligned}
$$




$$
\mathbb{R}_{s}\left(\theta_{0}, \omega_{0}, \omega\right)=i \frac{\omega_{0} \omega}{c^{2}} \frac{1+R_{s}\left(\theta_{0}, \omega_{0}\right)}{q^{>}+q^{<}} \chi_{s}^{\|} .
$$

In the latter expressions, the proper dimensionality is retrieved upon noting from Eq. (1) that $\left[\chi_{s}\right] \propto L[\chi]$. Recall that the normal Fresnel coefficients are given by

$$
\begin{gathered}
R_{p}\left(\theta_{0}, \omega_{0}\right)=\frac{\varepsilon(\omega) q_{0}^{>}-q_{0}^{<}}{\varepsilon(\omega) q_{0}^{>}+q_{0}^{<}}, \\
R_{s}\left(\theta_{0}, \omega_{0}\right)=\frac{q_{0}^{>}-q_{0}^{<}}{q_{0}^{>}+q_{0}^{<}},
\end{gathered}
$$

for $p$ and $s$ polarization, respectively, where the perpendicular wave-vector components are:

$$
\begin{array}{ll}
q_{0}^{>}=\left(\frac{\omega_{0}^{2}}{c^{2}}-K^{2}\right), & q_{0}^{<}=\left(\varepsilon\left(\omega_{0}\right) \frac{\omega_{0}^{2}}{c^{2}}-K^{2}\right), \\
q^{>}=\left(\frac{\omega^{2}}{c^{2}}-K^{2}\right), & q^{<}=\left(\varepsilon(\omega) \frac{\omega^{2}}{c^{2}}-K^{2}\right) .
\end{array}
$$

To illustrate the dependence on angle of incidence and frequency shift, the effective Fresnel coefficients for $\lambda_{0}$ $=2 \pi c / \omega_{0}=620 \mathrm{~nm}$ and a planar silver surface are plotted in Figs. 1(b)-1(d) (assuming $\chi_{s}^{\|}=\chi_{s}^{\perp}=1$ and Ag dielectric constants ${ }^{32}$ ). First, note the bright bands for $\mathbb{R}_{p}$ in the evanescent region [above the white curves, $\sin \theta=\left(\omega_{0} / \omega\right) \sin \theta_{0}$ $>1]$. These bands correspond to the surface-plasmon polariton (SP) dispersion relation (poles of $\mathrm{R}_{p}$ ) at $\omega$, retrieved for matching transverse wave-vector components $K$ provided that $\theta_{0}$ is large enough and the emission is sufficiently shifted to lower frequencies so that

$$
K_{0}=\left(\omega_{0} / c\right) \sin \theta_{0}=k_{S P}(\omega) \equiv(\omega / c)[\varepsilon(\omega) /(\varepsilon(\omega)+1)]^{1 / 2} .
$$

In essence, the molecular layer pumped at $\left(\theta_{0}, \omega_{0}\right)$ would be collectively exciting the SP at shifted frequency $\omega$, despite the fact that the surface is planar, thus leading to increased quenching. ${ }^{3}$ Second, note that proper angular dependences are retrieved with vanishing perpendicular and/or parallel components at normal and/or grazing incidence, as imposed by the polarization of the driving EM field and diagonal $\chi_{s}$. Third, to properly analyze the enhancement factors, singling out the impact of the metal surface from that of matching polarization and/or orientation, the effective Fresnel coefficients are renormalized by those for a freestanding dipole layer, leading to

$$
\begin{gathered}
\overline{\mathbb{R}}_{p}^{\perp}\left(\theta_{0}, \omega_{0}, \omega\right)=\mu\left(\omega, \omega_{0}\right)\left[1+R_{p}\left(\theta_{0}, \omega_{0}\right)\right]\left[1+R_{p}(\theta, \omega)\right], \\
\overline{\mathbb{R}}_{p}^{\|}\left(\theta_{0}, \omega_{0}, \omega\right)=\left[1-R_{p}\left(\theta_{0}, \omega_{0}\right)\right]\left[1-R_{p}(\theta, \omega)\right], \\
\overline{\mathbb{R}}_{s}\left(\theta_{0}, \omega_{0}, \omega\right)=\left[1+R_{s}\left(\theta_{0}, \omega_{0}\right)\right]\left[1+R_{s}(\theta, \omega)\right] .
\end{gathered}
$$

These enhancement factors are shown in Fig. 1(e) for fixed frequency shifts in the propagating region. ${ }^{33}$ As expected on a metal surface, the terms depending on tangential electric-field components are smaller, $\overline{\mathbb{R}}_{p}^{\|}$being mainly significant at large $\theta_{0}$. Nonetheless, $\overline{\mathbb{R}}_{p}^{\perp}$, stemming from the normal component, yields higher values (yet modest, $\left|\overline{\mathrm{R}}_{p}^{\perp}\right|^{2}$ $\propto\left|1+R_{p}\right|^{4} / 4 \lesssim 4$ for $\omega=\omega_{0}$ ), but simply due to normal interference between incident and reflected electric fields for grazing $\theta_{0}$. With regard to the pump frequency variation through the visible and near IR, and other noble metals, no significant qualitative differences are expected, and only slight quantitative variations appear due to the change in $\varepsilon(\omega)$.

\section{IMPLEMENTING THE COLLECTIVE MODEL IN THE SCATTERING FORMULATION}

We now proceed to exploit the model to investigate the emission and scattering of a molecular layer on complex nanostructured metal surfaces. The procedure should be as follows. First, the scattering problem is solved for the pump beam at $\omega_{0}$ illuminating the metal nanostructure, the molecular layer being passive at this stage. Then, the resulting total electric field (rather than the incident field) at the interface drives the emission of the surface molecular layer through the polarization vector [Eq. (1)]. Finally, another scattering problem for the metal nanostructure is solved at the emission frequency $\omega$, decoupled from that at $\omega_{0}$ by invoking the undepleted pump approximation, where the molecular layer emission plays the role of effective incident field. This procedure is independent of the scattering formulation being employed.

In particular, we make use of a rigorous scattering formulation based on the Green's theorem surface integral equations or generalized extinction theorem..$^{34,35}$ In this formulation, the electromagnetic field throughout the entire space of the corresponding scattering configuration can be expressed as surface integrals of the EM fields at the interfaces separating domains with different dielectric permittivities (nonmagnetic media are considered). Such surface EM fields play the role of source functions that, in general (no approximations), have to be determined by solving the system of surface integral equations obtained upon evaluating the above integral equations at the interface for the corresponding incident field, exploiting, in turn, the continuity conditions.

The formulation is rigorous from the standpoint of classical electrodynamics and has been successfully applied to study a variety of problems involving classical wave scattering from complex configurations. In addition, it is particularly suitable for the implementation of our theoretical model for the molecular layer emission, although other scattering formulations $^{29}$ could be employed within the proposed scheme. Essentially, two linear scattering problems are solved: First, the scattered EM field for the pump beam at $\omega_{0}$ is calculated. The resulting surface electric field is then introduced into the continuity conditions [Eqs. (2)] to drive the emission of the molecular layer at $\omega$. We thus exploit such modified continuity conditions in the scattering formulation at $\omega$, which give rise to an inhomogeneous term that plays the role of the incident beam at $\omega$ (recall that no incident beam as such does exist at $\omega$ ): this constitutes the second 
linear scattering problem to be solved, which, in turn, has no feedback onto the first scattering problem at $\omega_{0}$, since the undepleted pump approximation is invoked. In the case of a typical configuration involving a scattering volume $V$ of surface $S$ (cf. Sec. 1.3 in Ref. 35), the effective incident field at $\omega$ is given by

$$
\begin{aligned}
\mathbf{E}_{\mathrm{eff}}(\mathbf{r}, \omega)= & \int_{\mathbf{r}^{\prime} \in S^{-}} d \mathbf{r}^{\prime}\left[i \frac{\omega}{c}(\mathbf{n} \times \Delta \mathbf{H}) \cdot \mathcal{G}+(\mathbf{n} \times \Delta \mathbf{E}) \cdot(\nabla\right. \\
& \times \mathcal{G})], \quad \mathbf{r} \notin V
\end{aligned}
$$

which, in turn, depends on the surface electric field at $\omega_{0}$ through the continuity conditions [Eqs. (2)] for the tangential EM fields at $\omega$. The Green's dyadic inside $V$ is denoted by $\mathcal{G}$. Formally, this effective field enters in the formulation through the Green's theorem surface integral equation stemming from the volume integral inside $V\left(\mathbf{r}^{\prime} \in S^{-}\right)$, evaluated outside the scattering volume $(\mathbf{r} \notin V)$, upon exploiting the continuity conditions [Eqs. (2)] to relate the EM surface fields from inside in the integrand [cf. Eq. (17d) in Ref. 34 or Eq. (1.37) in Ref. 35] with those outside $V$. Equation (5) plays the role of the inhomogeneous term (source field at $\omega$ ).

\section{NUMERICAL CALCULATIONS: NANOANTENNAS AND SERS}

We now apply the above formulation to describe the molecular emission in two relevant configurations involving metal nanostructures, for which LSPR-mediated, surface EM field enhancements may occur: nanoparticles and disordered nanostructured surfaces. We restrict ourselves to bidimensional geometries for the sake of computational effort, which retain the main physics of the emission phenomena being studied (and are indeed rigorous in the case of, e.g., infinitely long nanowires and disordered gratings invariant along the $y$ direction). Moreover, assuming an incident beam with linear polarization ( $p$, or transversal magnetic, and $s$, or transversal electric), it has been shown that the three-dimensional vectorial formulation can be reduced to a two-dimensional scalar one for the nonzero $(y)$ component of the magnetic (electric) field for $p(s)$ polarization. The details on how to solve the linear scattering problem at $\omega_{0}$ can be found in Ref. 36, including the relation between the source fields at the surface with all the EM field components at, near, and far from the surface. In dealing with the molecular layer emission, the continuity conditions [Eq. (2)] and the effective incident field [Eq. (5)] can be further simplified; the details will be given elsewhere.

Let us now calculate the emission from two adjacent gold nanowires with rectangular cross section, similar to the nanoantennas investigated recently. ${ }^{23}$ The scattering cross section (SCS) of the dimer is shown in Fig. 2(a) as obtained through the surface integral scattering formulation mentioned above for different illumination angles and polarizations [cf. Fig. 2(b)]. A strong LSPR is observed for polarization parallel to the dimer axis at $\lambda=2 \pi c / \omega=665 \mathrm{~nm}$. Higherorder, multipolar resonances are not evident in Fig. 2(a), for
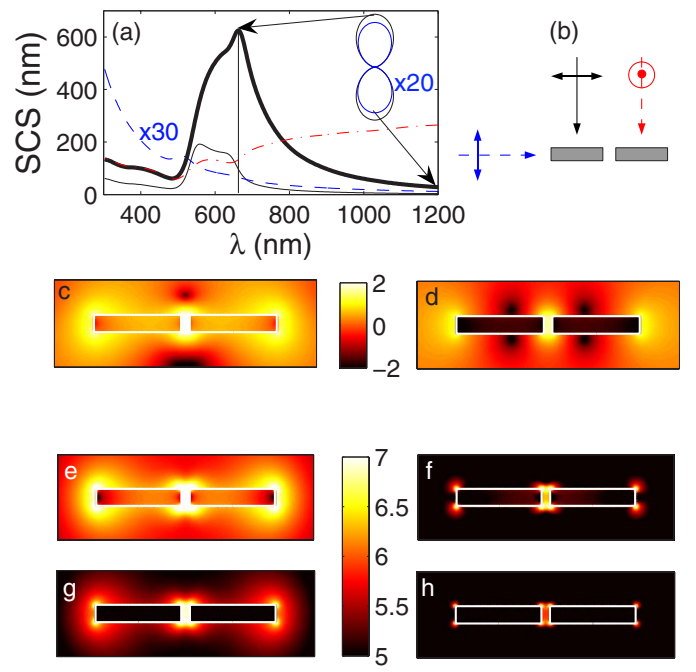

FIG. 2. (Color online) (a) Scattering cross sections for a dimer consisting of rectangular gold nanowires of $100 \mathrm{~nm}$ length and $20 \mathrm{~nm}$ width with a 10-nm-wide gap; incident angles and polarizations as shown in (b), the thin black curve being as the thick one but for a single nanowire. [(c) and (d)] Near-field intensity ( $\log _{10}$ scale, normalized to the incident field) at the pump frequency for incident direction and/or polarization perpendicular and/or parallel to the dimer axis: (c) on resonance $\left(\lambda_{0}=665 \mathrm{~nm}\right)$ and (c) off resonance $\left(\lambda_{0}=1.2 \mu \mathrm{m}\right)$; corresponding far-field patterns shown in the inset in (a). [(e)-(h)] Near-field intensity ( $\log _{10}$ scale, in a.u.) at the emission frequency for various pump and/or emission frequencies: (e) on-on, (f) on-off, (g) off-on, and (h) off-off.

they lie at frequencies higher than that of the onset of interband transitions, which strongly damp the SCS for small wavelengths. Incidentally, the rectangle's sharp corners have been replaced in the numerical calculations by rounded ones, ensuring that the results converge with decreasing radius. The impact of subwavelength corners in this scattering formulation has been thoroughly investigated (see, e.g., Ref. 37).

The LSPR at $\lambda=665 \mathrm{~nm}$ is then explored for various combinations of pump frequency and emission shift. Bear in mind that the effective incident field at the emission frequency, resulting from Eq. (5), though exhibits a complex pattern significantly different from that of the pump incident field, preserves polarization in the present two-dimensional configuration. The resulting near electric-field intensity maps at $\omega_{0}$ and $\omega$ are shown in Fig. 2 for (i) resonant pump and no shift [Fig. 2(e)]; (ii) resonant pump and nonzero shift, offresonance emission [Fig. 2(f)]; (iii) off-resonance pump and resonant emission [Fig. 2(g)]; and (iv) off-resonance pump and emission [Fig. 2(h)]. The near-field intensity patterns at the pump frequency are also shown for comparison at both the LSPR [Fig. 2(c)] and off-resonance $\lambda_{0}=1.2 \mu \mathrm{m}$ [Fig. 2(d)]. The LSPR near-field pattern corresponds to two coupled dipoles, one per nanowire, with antisymmetric charge distributions (not shown here) and large near-field enhancements at the gap in between, indeed similar to that reported for coupled nanorods ${ }^{21}$ this is corroborated by the far-field patterns [either half wave (resonance) or dipolar (off resonance), see the inset in Fig. 2(a)].

The near-field pattern resulting from emission at $\lambda$ $=665 \mathrm{~nm}$ [Figs. $2(\mathrm{e})$ and $2(\mathrm{~g})]$ is qualitatively similar to that 


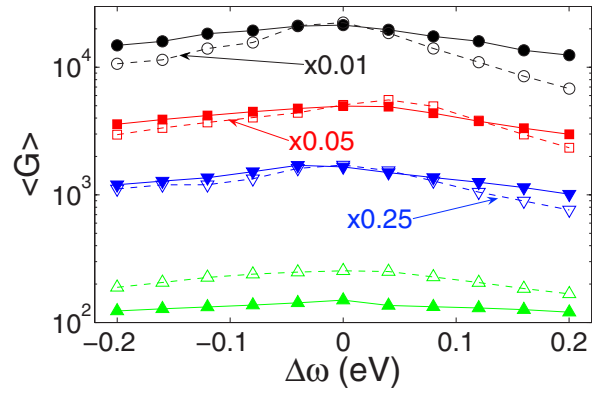

FIG. 3. (Color online) SERS enhancement factors (averaged over 200 realizations) as a function of the frequency shift for nanostructured silver substrates (pumped at $\omega_{0}=2 \mathrm{eV}, \lambda_{0}=620 \mathrm{~nm}$ ) with rms height $\delta$ and Gaussian correlation (correlation length $a$ ), calculated from our collective model $\langle G\rangle$ (solid curves, filled symbols) and from the approximation $\left\langle G^{(1)}\right\rangle$ (dashed curves, hollow symbols), with the latter rescaled by the shown factors: $a=51.4 \mathrm{~nm}$ with $\delta=5 a$ (circles), $\delta=2 a$ (squares), and $\delta=a$ (down triangles) and $a$ $=\delta=102.8 \mathrm{~nm}$ (up triangles).

of the LSPR near-field pattern at $\lambda_{0}=665 \mathrm{~nm}$ [Fig. 2(c)]; the same occurs for off-resonance emission at $\lambda_{0}=1.2 \mu \mathrm{m}$ [Figs. $2(\mathrm{f})$ and $2(\mathrm{~h})]$ in connection with off-resonance excitation at the same wavelength [Fig. 2(d)]. Quantitatively, near-field intensities at the dimer gap are substantially smaller for nonresonant $\omega_{0}$ or $\omega$, being minimum for both nonresonant $\omega_{0}$ and $\omega$.

The present model permits to yield true enhancement factors $\sigma$ of the collective dipolar emission from the resulting SCSs (rather than those inferred from average surface-field enhancements at the pump frequency $\left.\sigma_{S F}\right)$. Upon normalizing the SCS by that obtained for transparent dimers, $\sigma$ $\approx 160$ (on-on), 10 (on-off and off-on), and 1 (off-off), in contrast to the approximations $\sigma_{S F} \approx 6500$ (on-on), 1700 (onoff and off-on), and 480 (off-off).

We next exploit the emission model and scattering formulation to calculate the average properties of the Ramanshifted emission from a molecular layer on disordered nanostructured surfaces similar to those employed as SERS substrates. ${ }^{14}$ For the random surface profile, we employ an ensemble of Gaussian-correlated surfaces with Gaussian statistics, with nanoscale correlation lengths, which may yield strongly localized SP and large surface-field enhancements at the pump frequency. ${ }^{38}$ Recall that Raman scattering is highly incoherent, so that a position-dependent random phase is introduced into the surface Raman susceptibility in Eq. (2). Calculations are carried out for various ensembles of surfaceprofile realizations, from which the average SERS enhancement factors $\langle G\rangle$ (obviously, only of EM origin, leaving aside the charge-transfer enhancement mechanism) are obtained (see Fig. 3). In addition, the approximate SERS enhancement factor is obtained from $\left\langle G^{(1)}\right\rangle=\left\langle\left|E\left(\omega_{0}\right)\right|^{2}|E(\omega)|^{2}\right\rangle$, where $|E|^{2}$ is the local surface electric-field intensity, also calculated by means of the rigorous scattering formulation. Although both reproduce the decay as a function of the Raman frequency shift, the approximation $\left\langle G^{(1)}\right\rangle$ largely overestimates the enhancement factors even at $\omega=\omega_{0}$, with differences being actually larger precisely the rougher the surface. Moreover, the approximation $\left\langle G^{(0)}\right\rangle=\left\langle\left|E\left(\omega_{0}\right)\right|^{2}\right\rangle$ $\times\left\langle|E(\omega)|^{2}\right\rangle$, not shown, yields, in addition to similar quantitative discrepancies, a (misleading) monotonic increase with $\omega$. Thus, the collective model is needed to accurately calculate average SERS enhancement factors.

\section{CONCLUDING REMARKS}

In conclusion, the macroscopic EM emission from a surface molecular layer has been classically modeled through a polarization vector that is non-negligible only at the interface. Effective analytical Fresnel formulas for planar surfaces predict collective quenching when emission is redshifted. The proposed polarization vector can be introduced in different scattering formulations. In particular, the model has been incorporated into the scattering formulation based on Green's theorem surface integral equations, which is especially suitable to rigorously account for surface and near EM fields. The rigorously calculated surface electric field at the pump frequency drives the molecular emission at a shifted frequency through the model polarization vector, in such a way that an effective incident beam at the shifted emission frequency is obtained at the surface of complex nanostructures. The resulting formulation allows us to address a variety of collective molecular emission problems near complex surfaces. Note also that nonlinear terms may be easily included as well in the polarization vector [Eq. (1)] by means of a susceptibility tensor of higher rank, leading to the continuity conditions [Eqs. (2)], to be introduced into the scattering formulation through Eq. (5). Incidentally, it should be mentioned that fluorescence quenching is not directly addressed by this model and should be properly introduced in the corresponding decay rates; however, the dependence on the distance to the metal surface could be accounted for in the model and calculations by introducing a thin layer playing the role of a spacer.

We have thus investigated collective EM processes mediated by surface-plasmon excitation in both the spontaneous emission near metal nanoantennas and SERS close to disordered nanostructured metal substrates by means of near-field and far-field patterns at emission frequencies. With regard to average SERS factors, proper (rather than inferred) enhancement factors are given that reveal that typical approximations tend to overestimate enhancement factors and to underestimate qualitatively and quantitatively frequency-shift impact. Our model and scattering formulation provide an accurate classical EM tool to study a wealth of surface molecular (or thin layer) emission processes (fluorescence, photoluminescence, Raman, etc.) near nanostructures where collective and/or surface plasmon (or other resonant phenomena) excitation play a role, with potential applications in nanophotonics and/or biosensing.

\section{ACKNOWLEDGMENTS}

This work was supported in part by the Spanish "Ministerio de Educación y Ciencia" (Grant Nos. FIS2006-07894 and FIS2004-0108) and "Comunidad de Madrid" (Grant No. S-0505/TIC-0191 and V.G.'s Ph.D. scholarship). 
*Author to whom correspondence should be addressed. Electronic address: j.sanchez@iem.cfmac.csic.es

${ }^{1}$ F. J. García-Vidal and J. B. Pendry, Phys. Rev. Lett. 77, 1163 (1996).

${ }^{2}$ M. Leyva-Lucero, E. R. Mendez, T. A. Leskova, A. A. Maradudin, and J. Q. Lu, Opt. Lett. 21, 1809 (1996).

${ }^{3}$ W. L. Barnes, J. Mod. Opt. 45, 661 (1998).

${ }^{4}$ S. Nie and S. R. Emory, Science 275, 1102 (1997).

${ }^{5}$ K. Kneipp, Y. Wang, H. Kneipp, L. T. Perelman, I. Itzkan, R. R. Dasari, and M. S. Feld, Phys. Rev. Lett. 78, 1667 (1997).

${ }^{6} \mathrm{H}$. Xu, E. J. Bjerneld, M. Käll, and L. Börjesson, Phys. Rev. Lett. 83, 4357 (1999).

${ }^{7}$ M. Leyva-Lucero, E. R. Méndez, T. A. Leskova, A. A. Maradudin, and J. Q. Lu, Opt. Commun. 161, 79 (1999).

${ }^{8}$ V. M. Shalaev, Phys. Rep. 272, 61 (1996); A. K. Sarychev and V. M. Shalaev, ibid. 335, 275 (2000).

${ }^{9}$ C. I. Valencia, E. R. Méndez, and B. S. Mendoza, J. Opt. Soc. Am. B 20, 2150 (2003).

${ }^{10}$ W. L. Barnes, A. Dereux, and T. W. Ebbesen, Nature (London) 424, 824 (2003).

${ }^{11}$ P. Andrew and W. L. Barnes, Science 306, 1002 (2004).

${ }^{12}$ K. Okamoto, I. Niki, A. Shvartser, Y. Narukawa, T. Mukai, and A. Scherer, Nat. Mater. 3, 601 (2004).

${ }^{13}$ R. Paiella, Appl. Phys. Lett. 87, 111104 (2005).

${ }^{14}$ C. L. Haynes, A. D. McFarland, and R. P. Van Duyne, Anal. Chem. 77, 338A (2005).

${ }^{15}$ P. Anger, P. Bharadwaj, and L. Novotny, Phys. Rev. Lett. 96, 113002 (2006).

${ }^{16}$ S. Kühn, U. Håkanson, L. Rogobete, and V. Sandoghdar, Phys. Rev. Lett. 97, 017402 (2006).

${ }^{17}$ J. Kottmann, O. Martin, D. Smith, and S. Schultz, Opt. Express 6, 213 (2000).

${ }^{18}$ E. Hao and G. C. Schatz, J. Chem. Phys. 120, 357 (2004).

${ }^{19}$ Mark I. Stockman, Phys. Rev. Lett. 93, 137404 (2004).

${ }^{20}$ L. A. Blanco and F. J. García de Abajo, Opt. Lett. 29, 1494 (2004).

${ }^{21}$ J. Aizpurua, G. W. Bryant, L. J. Richter, F. J. García de Abajo, B.
K. Kelley, and T. Mallouk, Phys. Rev. B 71, 235420 (2005).

${ }^{22}$ U. Hohenester and J. Krenn, Phys. Rev. B 72, 195429 (2005); A. Hohenau, J. R. Krenn, J. Beermann, S. I. Bozhevolnyi, S. G. Rodrigo, L. Martín-Moreno, and F. García-Vidal, ibid. 73, 155404 (2006)

${ }^{23}$ P. Mühlschlegel, H.-J. Eisler, O. J. F. Martin, B. Hecht, and D. W. Pohl, Science 308, 1607 (2005).

${ }^{24}$ T. Kalkbrenner, U. Håkanson, A. Schädle, S. Burger, C. Henkel, and V. Sandoghdar, Phys. Rev. Lett. 95, 200801 (2005).

${ }^{25}$ P. J. Schuck, D. P. Fromm, A. Sundaramurthy, G. S. Kino, and W. E. Moerner, Phys. Rev. Lett. 94, 017402 (2005).

${ }^{26}$ C. L. Nehl, H. Liao, and J. Hafner, Nano Lett. 6, 683 (2006).

${ }^{27}$ J. J. Greffet, Science 308, 1651 (2005).

${ }^{28}$ R. R. Chance, A. Prock, and R. Silbey, Adv. Chem. Phys. 37, 1 (1978).

${ }^{29}$ Ch. Girard and A. Dereux, Rep. Prog. Phys. 59, 657 (1996).

${ }^{30}$ For a simple noninteracting dipole model, for instance $\chi$, should acquire the form of a polarizability times the molecular surface density, rather than volume density.

${ }^{31}$ M. Born and E. Wolf, Principles of Optics, 6th ed. (Pergamon, Oxford, 1980), pp. 4-6.

${ }^{32}$ Handbook of Optical Constants of Solids, edited by E. D. Palik (Academic, Orlando, 1985).

${ }^{33}$ Bear in mind that the Fresnel coefficients in the evanescent region cannot be considered as enhancement factors since the corresponding emitted waves do not contribute to the radiative field.

${ }^{34}$ E. Wolf, in Coherence and Quantum Optics, edited by L. Mandel and E. Wolf (Plenum, New York, 1973), pp. 339-357.

${ }^{35} \mathrm{M}$. Nieto-Vesperinas, Diffraction and Scattering in Physical Optics (Wiley, New York, 1991).

${ }^{36}$ J. A. Sánchez-Gil, J. V. García-Ramos, and E. R. Méndez, Phys. Rev. B 62, 10515 (2000).

${ }^{37}$ A. Mendoza-Suárez and E. R. Méndez, Appl. Opt. 36, 3521 (1997).

${ }^{38}$ J. A. Sánchez-Gil, J. V. García-Ramos, and E. R. Méndez, Opt. Lett. 26, 1286 (2001); Opt. Express 10, 879 (2002). 